\title{
Uncemented three-dimensional-printed prosthetic replacement for giant cell tumor of distal radius: a new design of prosthesis and surgical techniques
}

This article was published in the following Dove Press journal: Cancer Management and Research

\section{Minxun Lu* \\ Li Min* \\ Cong Xiao \\ Yongjiang Li \\ Yi Luo \\ Yong Zhou \\ Wenli Zhang \\ Chongqi Tu}

Department of Orthopedics, West China Hospital, Sichuan University, Chengdu, Sichuan, People's Republic of China

*These authors contributed equally to this work
Correspondence: Chongqi Tu Department of Orthopedics, West China Hospital, Sichuan University, No. 37 Guoxue Street, Chengdu 61004I, Sichuan, People's Republic of China Tel +86 I82 80060169 Fax +8602885422454

Email Tuchongqi@yeah.net
Introduction: Currently, it is challenging to treat giant cell tumor (GCT) of distal radius. For Campanacci grade III or recurrent GCTs, en bloc resection has been accepted as a better treatment option. Although numerous methods are available for reconstruction, all of them have some limitations in joint function and complications. In this study, our aims were to treat the GCT of distal radius with uncemented three-dimensional (3D)-printed prosthesis and to present and evaluate the surgical techniques and short-term outcomes.

Methods: Between September 2015 and March 2017, 11 patients with distal radius GCTs were treated with personalized uncemented 3D-printed prosthesis. The preoperative/postoperative pain, range of motion, and grip strengths of all patients were evaluated. Oncological results, complications, and degenerative changes in the wrist joint were evaluated. Functional outcomes were assessed according to the disabilities of the arm, shoulder, and hand (DASH) questionnaire and Mayo wrist scoring systems.

Results: The average follow-up was 14.45 months (range, $8-18$ months). There was a significant decrease in the mean postoperative visual analog scale score (2.33) compared with the preoperative score $(5.22 ; p<0.001)$. The mean DASH score and Mayo wrist score of the wrist joint function were 18.7 and 72, respectively. There was no local recurrence or lung metastasis. No complication associated with prosthesis was observed, including aseptic loosening, subluxation, and breakage. Joint space narrowing, or disuse osteoporosis, was also not found in all cases.

Conclusion: En bloc resection and reconstruction with a personalized uncemented 3D-printed prosthesis can be alternative options to treat Campanacci grade III or recurrent GCTs of distal radius and can result in short-term oncologic salvage, good postoperative function, and low complication rate. However, a long-term follow-up is required to determine the outcome.

Keywords: rapid prototyping, computer-aided design, cementless, uncemented, polyethylene liner

\section{Introduction}

Giant cell tumor (GCT) of bone is a potentially aggressive lesion with a relatively high recurrence rate after primary treatment. ${ }^{1}$ GCTs mostly occur between the ages of 20 and 40 years. ${ }^{2}$ Approximately $10 \%$ of GCTs are located at the distal radius, ${ }^{3,4}$ which is the third most common site affected. ${ }^{4}$ Intralesional curettage and cementation are regular treatments for patients with Campanacci grade I or II tumors. Considering the

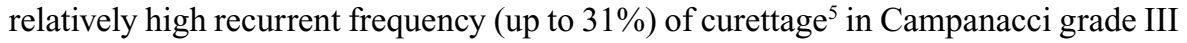
or recurrent tumors, ${ }^{4,6,7}$ en bloc resection has been accepted as the standard treatment. ${ }^{8}$ However, postoperative wrist function may be compromised. ${ }^{8}$

Previously, numerous procedures were applied for the reconstruction of distal radius, including wrist joint arthrodesis, ${ }^{9}$ nonvascularized ${ }^{10}$ or vascularized fibula 
autograft, ${ }^{11}$ osteoarticular allografts, ${ }^{12}$ and prosthetic reconstruction. ${ }^{13-18}$ Although these procedures have been reported to result in reasonable functional outcomes with regard to patient satisfaction or the ability to return to a normal life, they have their own limitations. ${ }^{19}$ The nonvascularized or vascularized fibula autografts were reported to have potential complications, including nonunion, delayed union, graft fracture, wrist joint subluxation, and donor site morbidity. Reconstruction using osteoarticular allograft seems promising. However, limited resource of allograft, nonuniformity, and mismatching between graft and palm bone may greatly limit this technique. ${ }^{12,20,21}$ The most common complications of prosthetic reconstruction are radiocarpal joint instability and stiffness because of the incompatible contact area. ${ }^{13-18,22}$ There may be some disadvantages in previous designing of prosthesis to be optimized, ${ }^{13-18,22,23}$ including low matching degree between prosthesis and bone, the high rate of aseptic loosing caused by cemented implantation, and the improper usage of polyethylene spacer or liner. We proposed that a custom-made, uncemented three-dimensional (3D)-printed prosthesis with a best-fit articular surface would be a better choice for designing. However, there is no related study regarding custom-made uncemented 3D-printed prosthetic reconstruction for tumor-induced defect of distal radius. Therefore, we retrospectively evaluated custom-made, uncemented 3D-printed prosthetic reconstruction in a group of patients with grade III or recurrent GCTs of the distal radius.

\section{Methods}

\section{Patients}

Between September 2015 and March 2017, 11 patients with GCTs of the distal radius received en bloc resection and uncemented 3D-printed prosthetic reconstruction in our institution. All the patients had Campanacci grade III or recurrent GCTs without metastasis (Figure 1). Of the 11 patients, 6 were male and 5 were female. The average age of the patients at the time of admission was 38 years (range, 31-45 years). The two recurrent patients were previously treated with extended curettage, local adjuvant therapy, and cementation.

All the patients had X-ray, 3D computed tomography (3D-CT), magnetic resonance imaging, bone scintigraphy, and preoperative biopsy. The pain, range of motion (ROM), and grip strength of the involved wrist were also assessed preoperatively. The pain at rest was evaluated according to a visual analog scale (VAS) in which 0 represents "no pain" and 10 represents "the worst pain imaginable." The ROM was

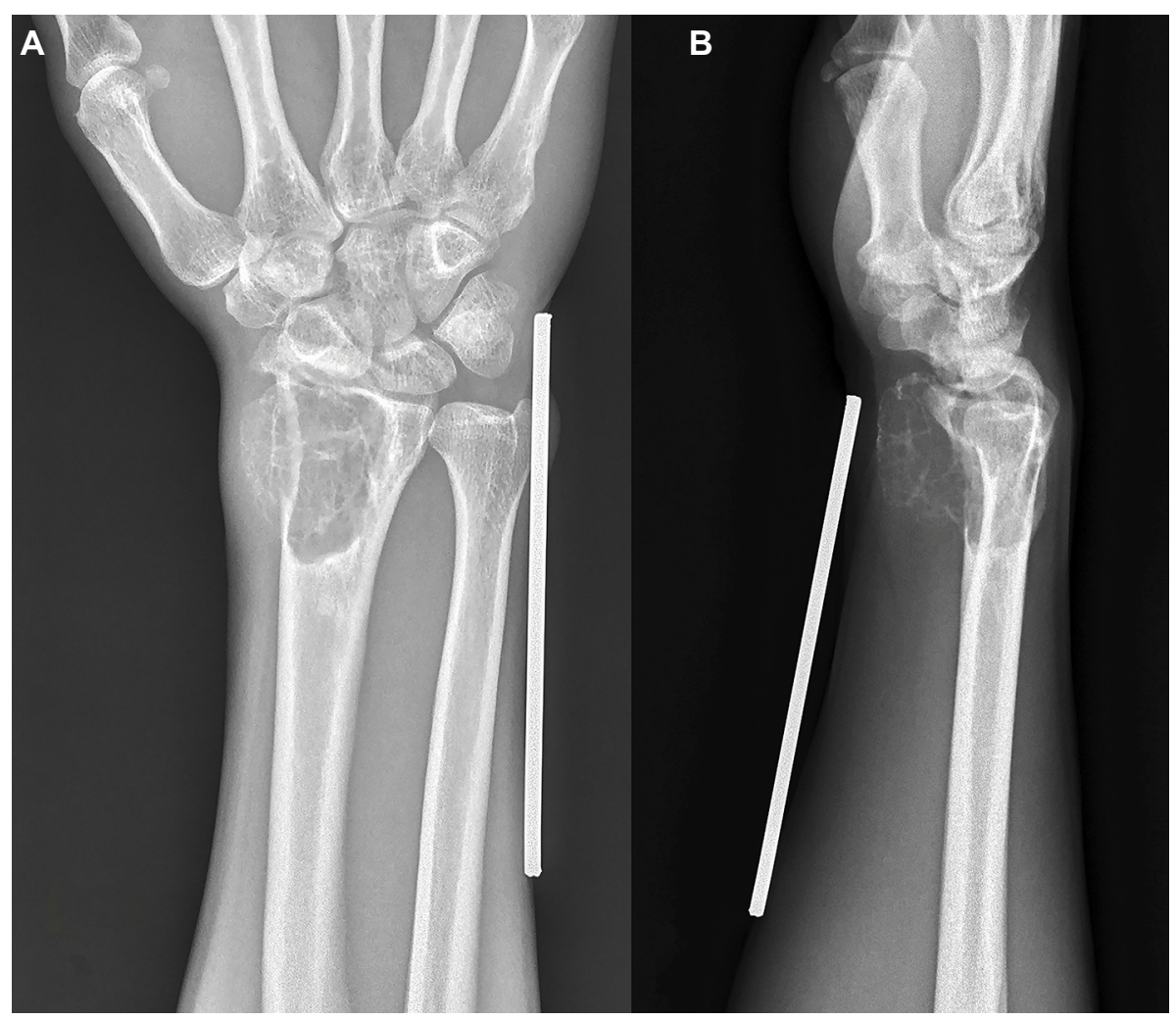

Figure I (A) Posteroanterior radiograph of a Campanacci grade III GCTs of the distal radius; (B) lateral radiograph of a Campanacci grade III GCTs. Abbreviation: GCT, giant cell tumor. 
recorded using a goniometer. Grip strength was measured using a hydraulic hand dynamometer (North Coast Medical, Inc., Gilroy, CA, USA). The patients completed Chinese validated translations of the disabilities of the arm, shoulder, and hand (DASH) questionnaire. ${ }^{24}$ In addition, the Mayo wrist score ${ }^{25}$ was also calculated for each patient. After the surgery, follow-up was performed every 3 months during the first year and every 6 months thereafter. The VAS, ROM, grip strength, DASH score, and Mayo wrist score were assessed at each follow-up visit to verify the outcomes of uncemented 3D-printed prosthetic reconstruction. Meanwhile, oncological outcomes, complications, and degenerative changes in wrist joint including joint space narrowing and disuse osteoporosis of carpus were also radiographically assessed.

This retrospective study was approved and monitored by the Ethical Committee of West China Hospital, Sichuan University, Chengdu, People's Republic of China, and was performed in accordance with the ethical standards of the 1964 Declaration of Helsinki. Written informed consent to participate in this study was obtained from all the patients. The patients balanced the risks and benefits of the uncemented 3D-printed prosthetic arthroplasty before signing the informed consent. All the patients gave permission to publish the images.

\section{Prosthesis design and fabrication}

All prostheses were individually designed by our clinical team and fabricated by Chunli Co., Ltd., Tongzhou, Beijing, People's Republic of China. Imitating normal radius appearance is the major principle for our designing. Building 3D computer models for the distal radius, distal ulnar, and palmar bones of the affected and contralateral normal radius for these patients was the first step, by importing the data from the 3D-CT scan into Mimics V17.0 software (Materialise Corp., Leuven, Belgium). Mirroring of the contralateral normal distal radius was done to obtain a preliminary model of the prosthesis. The high matching suitability for the carpus and ulnar head of the affected side was ensured using the mirroring process. In order to protect the cartilage of the palmar bone, ultrahigh-molecular-weight polyethylene (Orthoplastics Ltd., Lancashire, UK) was used to fabricate the articular surface of the prosthesis (Figure 2). To assemble the two parts tightly, a specialized polyethylene lock structure was created on the proximal plane of polyethylene liner. Ten repairing pores were designed to reconstruct the radiocarpal joint and the distal ulnoradial joint by suturing the surrounding soft tissue (Figure 3).

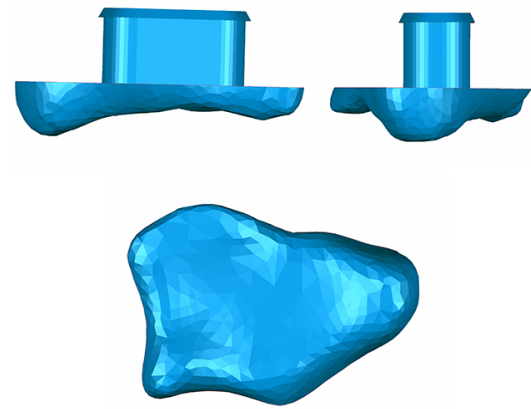

Figure 2 Anterioposterior view, lateral view, and vertical view of 3D model of polyethylene liner.

Abbreviation: 3D, three-dimensional.
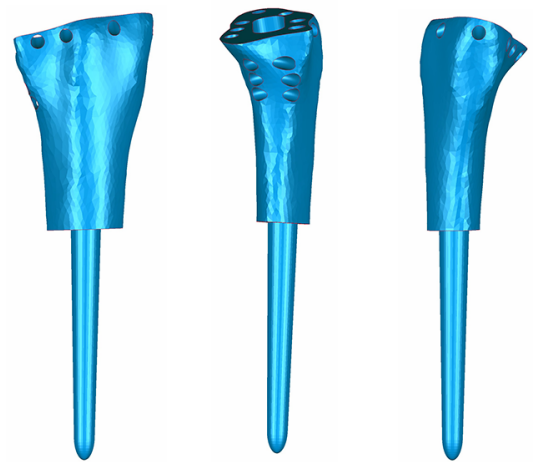

Figure 3 Anterioposterior view, left view, and right view of 3D model of titanium part (shaft and stem).

Abbreviation: 3D, three-dimensional.

Computerized numerical control engraving technology was applied for the manufacture of the ultrahigh-molecularweight polyethylene liner. The rest part of the prosthesis, including the shaft and stem, was fabricated by electron beam melting technology (ARCAM Q10, Mölndal, Sweden). After completing the above procedures, all prostheses were coated with hydroxyapatite (Figure 4).

\section{Surgical technique}

All surgeries were performed by the same senior surgeon (Chongqi Tu). The GCTs of the distal radius were exposed through a dorsal approach under general anesthesia. En bloc resection was done $1.5 \mathrm{~cm}$ away from the tumor border (Figure 5). During the operation, four patients needed to have the palmar ligaments and tendons cut, and the joint capsule required significant removal because of the invasion of the lesion. Meanwhile, we successfully retained all radiocarpal ligaments, tendons, and the joint capsule in seven patients with no lesion invasion. When implanting the prosthesis, the rotation could be controlled depending upon the crest of the prosthesis. The stem was press-fit inserted into the reamed radius canal. The next procedure was to reconstruct the distal 


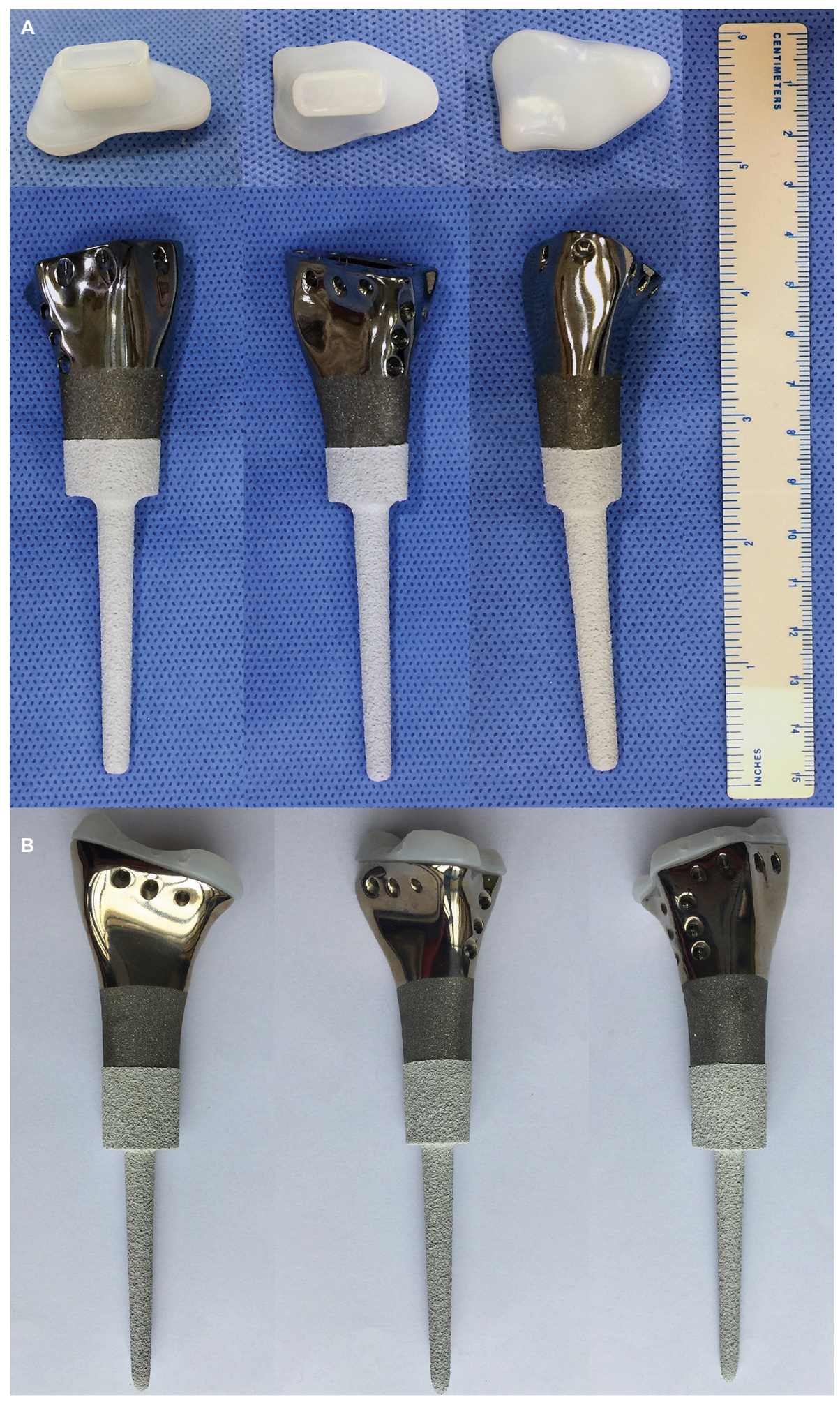

Figure 4 (A) Components of prosthesis; (B) endoprosthesis with HA coating. Abbreviation: HA, hydroxyapatite.

radioulnar joint and the radiocarpal joint. The nonabsorbable suture (Ethibond ${ }^{\mathrm{TM}}$ size 2; Johnson \& Johnson, Ltd., New Brunswick, NJ, USA) was used to sew up the remaining soft tissue, such as palmar ligaments, tendons, triangular fibrocartilage complex, and joint capsule, to prosthesis pores to enhance joint stability (Figure 6). 


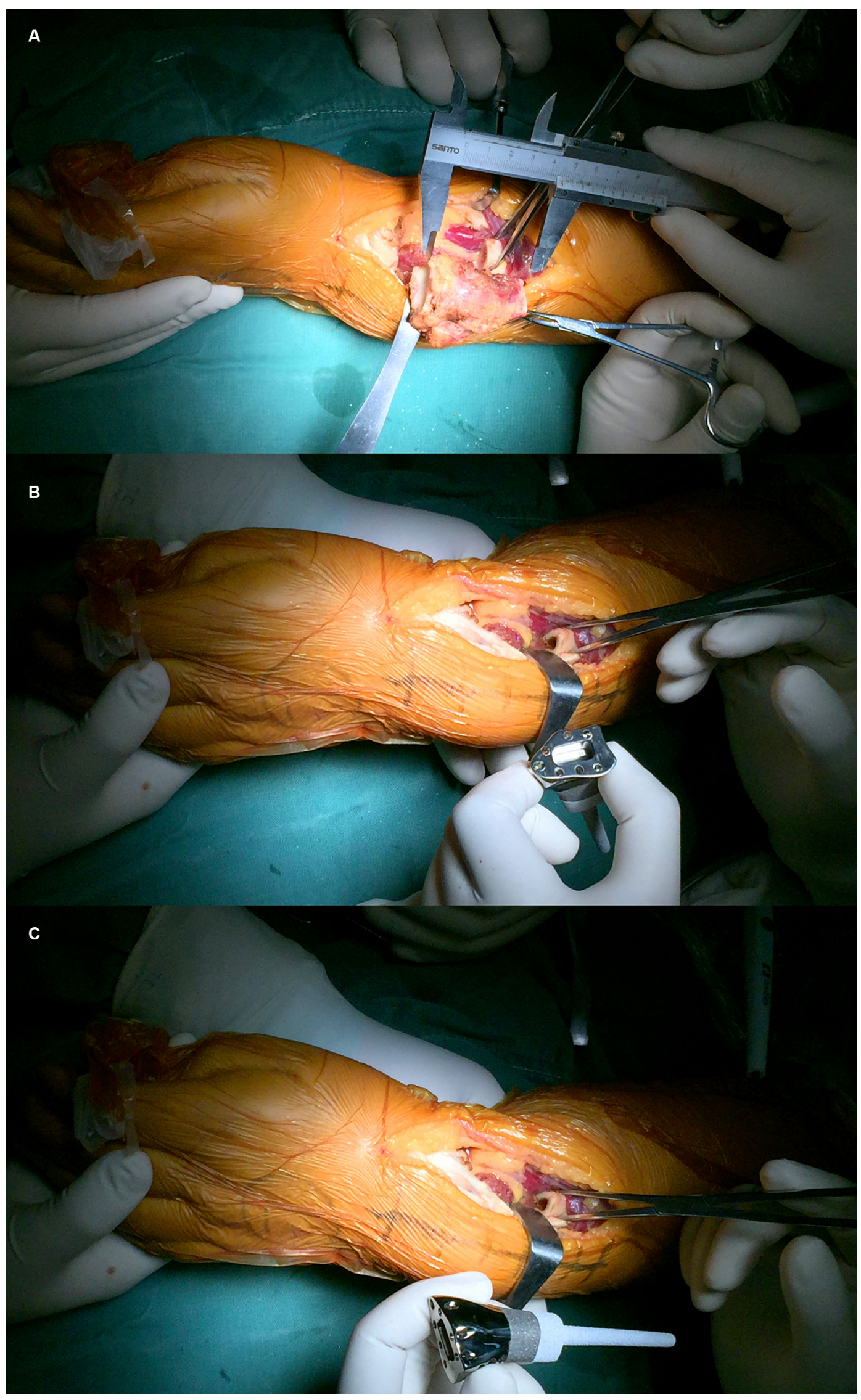

Figure 5 Intraoperative picture indicating the resection of the distal radius via a dorsal approach.

Notes: (A) Measuring length of tumor defect. (B) Removal of tumor. (C) Preliminary evaluation of implantation feasibility. 


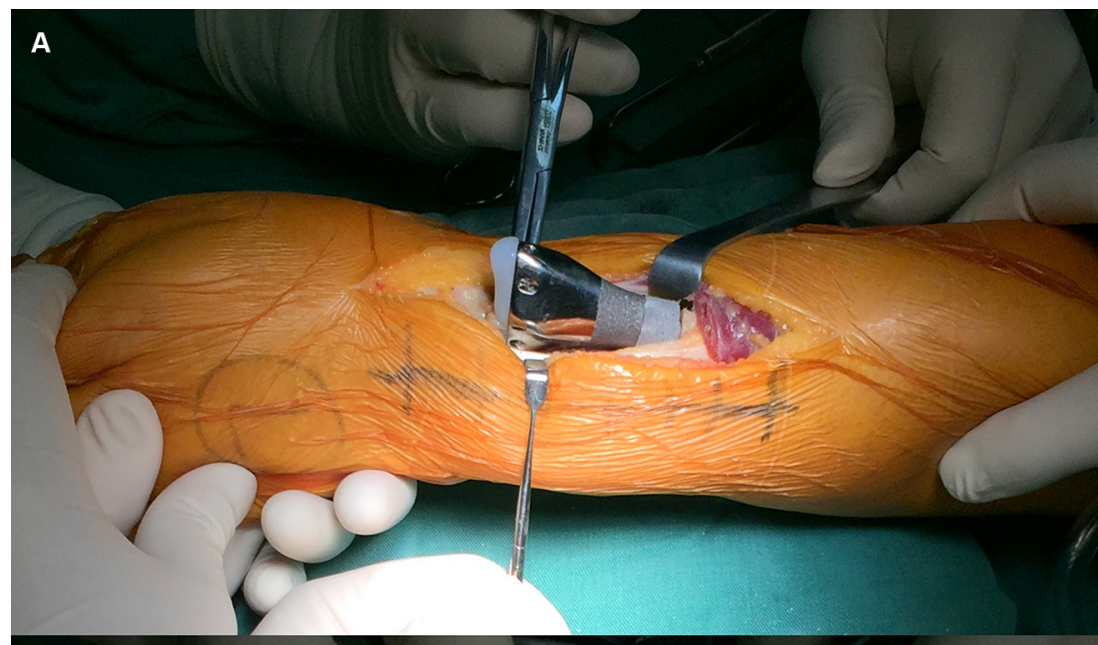

B
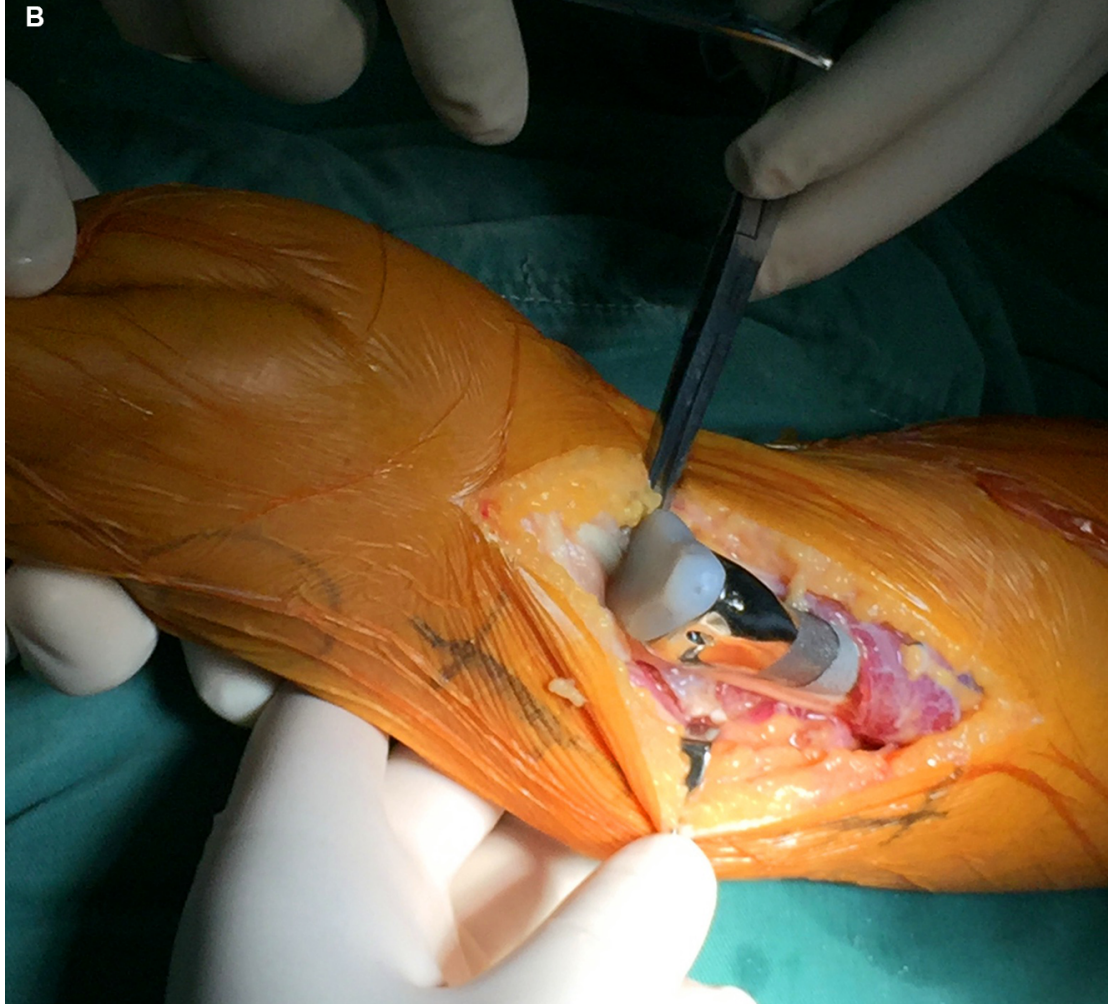

c

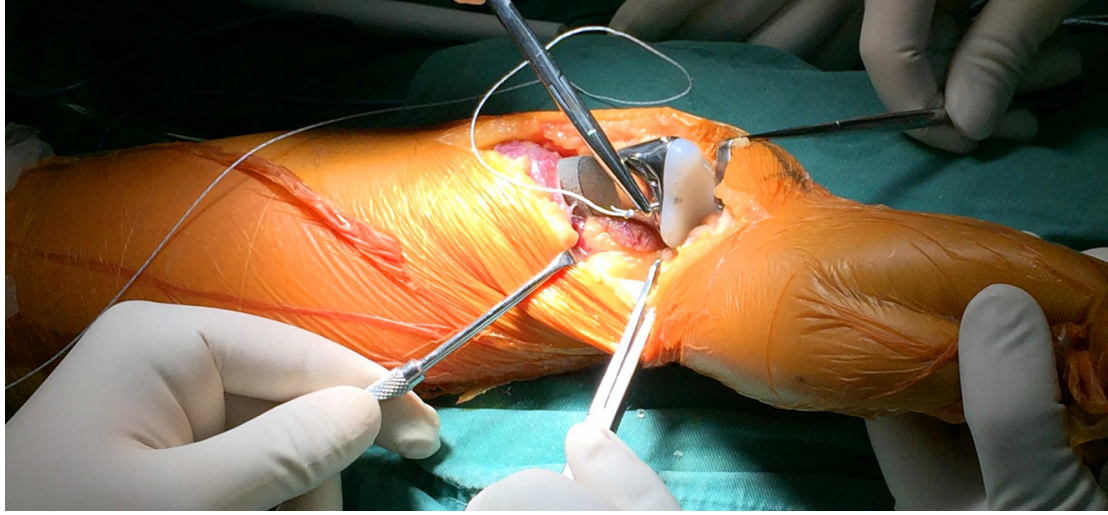

Figure 6 Replacement and repair wrist joint and distal ulnoradial joint.

Notes: (A) Implanting was completed. (B) The length of prosthesis was suitable for tumor-induced defect. (C) Repairing the joint with nonabsorbable suture. 


\section{Postoperative management}

In four cases, the affected limb of patients who underwent massive resection of palmer ligaments, tendons, and joint capsule due to tumor invasion was immobilized in an aboveelbow cast for 4 weeks to stabilize the joint by promoting scarring of periprosthetic tissue. Wrist exercises were then performed to activate the ROM. By contrast, for rest cases, in which most of joint capsule was retained, the postoperative exercise was timely carried out to achieve more flexible ROM. We gradually increased the intensity of exercise according to each patient's tolerance and recovery progress.

\section{Statistical analysis}

Continuous data are represented as the mean \pm standard deviation. Student's $t$-test was used to compare continuous variables. SPSS software (Version 20; IBM Corporation, Armonk, NY, USA) was used for statistical analysis. A $p<0.05$ was considered statistically significant.

\section{Results}

All the patients were followed up for a mean duration of 14.45 months (range, 8-18 months; Table 1). Before the surgery, all of them complained of pain, and their mean VAS score was 5.22 (range, 4-7). After the surgery, seven patients had obvious relief from pain, while four patients continued to suffer from moderate pain. At the last follow-up, the mean VAS score was 2.33 (range, $1-3 ; p<0.0001$ ). The average ROMs measured at the last follow-up were $61.55^{\circ}$ (range, $40^{\circ}-78^{\circ}$ ) of dorsiflexion, $30^{\circ}$ (range, $20^{\circ}-40^{\circ}$ ) of palmar flexion, $59.95^{\circ}$ (range, $30^{\circ}-80^{\circ}$ ) of supination, and $57.27^{\circ}$ (range, $30^{\circ}-75^{\circ}$ ) of pronation, respectively. Compared with preoperative ROM, there was a significant improvement in ROM, except for palmar flexion, after surgery $(p<0.05)$. The average preoperative grip strength was $17 \mathrm{mmHg}$ (range,
9-31 $\mathrm{mmHg}$ ), while postoperatively it was $23.6 \mathrm{mmHg}$ (range, $18-30 \mathrm{mmHg})$ in the affected extremity $(p<0.05)$. The mean DASH score significantly decreased by 11 (from 29.7 to $18.7 ; p<0.05)$. In addition, the postoperative Mayo wrist score improved from 28.7 to $72(p<0.0001)$. During the follow-up period, there was no death, recurrence of tumor, metastasis, or amputation. In addition, no degenerative changes or complications associated with prosthetic replacement, such as aseptic loosening, subluxation, or breakage, were observed (Figure 7). Table 2 summarizes functional outcomes and degenerative changes. Figure 8 shows a typical case.

\section{Discussion}

Campanacci grade III GCT and recurrent GCT of the distal radius are both locally aggressive lesions with a relatively high recurrence rate. The main therapeutic aims are the following: resecting the tumor completely, decreasing the rate of local recurrence, and preserving the maximum possible wrist joint function. In order to minimize the chance of recurrence, en bloc resection has been widely accepted for Campanacci grade III GCTs. ${ }^{20,26}$ Because of the limitations and disadvantages of allograft/autograft reconstruction, prosthetic arthroplasty has become mainstream. Prosthetic reconstruction after resection has been previously reported with reasonable outcomes. ${ }^{13-18,22}$ However, the prosthetic reconstruction reported in these studies resulted in unsatisfactory ROM and a high rate of complications. We performed arthroplasty using custom-made uncemented 3D-printed prosthetics in 11 patients with Campanacci grade III or recurrent GCTs of the distal radius. We found that postoperative function was satisfactory, and no complications occurred.

In our study, we observed obvious improvements in ROM and a decreased complication rate compared with previous studies of prosthetic replacement for the wrist joint. Since

Table I Patients' basic characteristics

\begin{tabular}{|c|c|c|c|c|c|c|c|}
\hline Patient & Age (years) & Gender & Side & $\begin{array}{l}\text { Campanacci } \\
\text { grade }\end{array}$ & Initial treatment & $\begin{array}{l}\text { Length of resected } \\
\text { bone }(\mathrm{cm})\end{array}$ & $\begin{array}{l}\text { Follow-up } \\
\text { (months) }\end{array}$ \\
\hline I & 31 & Female & Left & III & Prosthetic arthroplasty & 4.20 & 18.00 \\
\hline 2 & 42 & Male & Right & Recurrent & Curettage and cementation & 5.50 & 16.00 \\
\hline 3 & 45 & Male & Left & III & Prosthetic arthroplasty & 5.00 & 11.00 \\
\hline 4 & 32 & Female & Left & III & Prosthetic arthroplasty & 4.00 & 8.00 \\
\hline 5 & 37 & Female & Right & III & Prosthetic arthroplasty & 3.80 & 16.00 \\
\hline 6 & 38 & Male & Left & Recurrent & Curettage and cementation & 6.00 & 12.00 \\
\hline 7 & $4 I$ & Female & Right & III & Prosthetic arthroplasty & 5.50 & 17.00 \\
\hline 8 & 35 & Female & Right & III & Prosthetic arthroplasty & 4.00 & 18.00 \\
\hline 9 & 45 & Male & Left & III & Prosthetic arthroplasty & 4.50 & 14.00 \\
\hline 10 & 34 & Male & Right & III & Prosthetic arthroplasty & 4.80 & 17.00 \\
\hline II & 38 & Male & Right & III & Prosthetic arthroplasty & 5.00 & 12.00 \\
\hline Mean & 38 & & & & & 4.75 & 14.45 \\
\hline
\end{tabular}




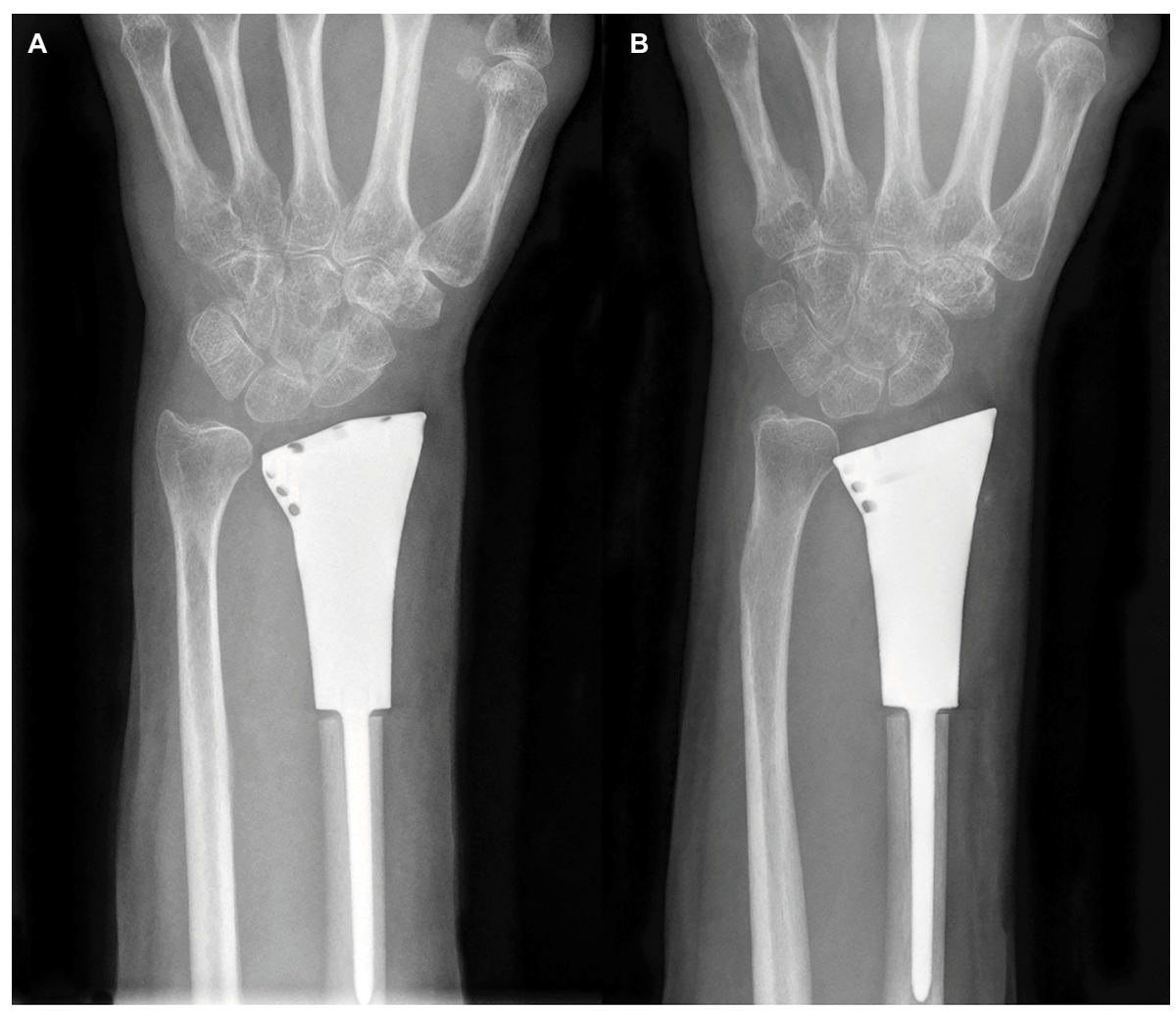

Figure 7 (A) One-month postsurgical posteroanterior radiographs; (B) posteroanterior radiographs also were obtained 10 months after surgery. There were no significant degenerative changes.

Gold's report in $1957^{23}$ (Table 3), many types of cemented prostheses have been used in the reconstruction of the wrist joint after tumor resection. ${ }^{13-18,22}$ In Natarajan et al's study, ${ }^{16} 16$ patients with distal radial GCTs were received custom-made cemented megaprosthesis reconstruction during the period 1995-2006. After an average of 78 months of follow-up, the mean flexion and extension were just $25^{\circ}$ and $20^{\circ}$, respectively. In addition, complications occurred in six patients, including aseptic loosening, infection, and skin flap necrosis. Hatano et $\mathrm{l}^{15}$ presented two cases of alumina ceramic prosthetic replacement for massive bone defects of the distal radius. Both patients had degenerative changes to the wrist $>10$ years after the procedure. Meanwhile, aseptic loosing occurred in both. At the last follow-up, the average flexion and extension were only $15^{\circ}$ and $30^{\circ}$, respectively. In 2013, Hariri et a ${ }^{17}$ reported the case of a 72-year-old woman in whom a mega wrist polyethylene spacer prosthesis was used to reconstruct the distal radius after en bloc resection of a GCT. Three years after the surgery, the prosthesis was well aligned, but with dorsal subluxation of the ulnar head. The similar type of prosthesis was used in Damert et al's study. ${ }^{18}$ The extension achieved was only $10^{\circ}$. Recently, Zhang et al ${ }^{13}$ and Wang et $\mathrm{al}^{14}$ implanted a custom-made prosthesis (LDK Co., Ltd., Haidian, Beijing, People's Republic of China) with a polyethylene liner to reconstruct the wrist joint following the en bloc resection. The average flexion and extension just reached $20^{\circ}-30^{\circ}$ and $20^{\circ}-40.9^{\circ}$, respectively. Wang et $\mathrm{al}^{14}$ observed a high proportion of complications ( 6 of $10 ; 60 \%$ ), including aseptic loosening, wrist subluxation, and joint degeneration. Contrary to the previous reports, we found that postoperative dorsiflexion could reach $61.55^{\circ}$ on average and $70^{\circ}$ in the majority of cases in this study because of the anatomical shape of our prosthesis. The average flexion could just reach $30^{\circ}$. The reason for this is that the retained soft tissue of the dorsal side was sutured more tightly than the palmar side to avoid the dislocation of the prosthesis. In addition, we observed no complications associated with the prosthesis, including subluxation, loosening, or breakage, at the final follow-up. Mean grip strength was $23.6 \mathrm{mmHg}$, which is in keeping with other reports of postoperative grip strength. ${ }^{13,15}$ Although follow-up is not yet sufficient to verify the long-term rate of complications, our results are considered indicative, as most complications occur within the first 2 years after surgery.

Besides the shape and thickness of the liner, a suitable length of the shaft including the crest and the use of hydroxyapatite coating would be the main factors that result in good function with a low chance of complications. First, the shape 


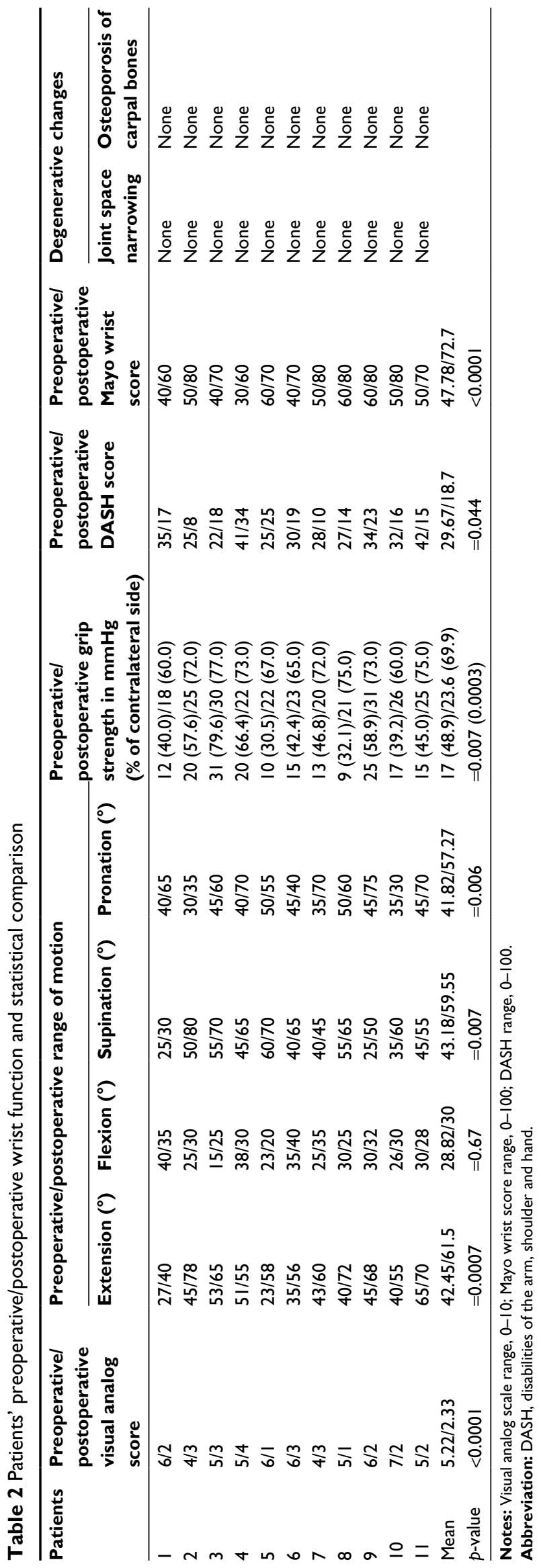

of the polyethylene liner is an essential factor for achieving a reasonable ROM. Although a polyethylene liner used in two previous studies ${ }^{13,14}$ had some impacts on preventing cartilage degeneration of the carpus, the shape and size were not suitable for the palmar and ulnar bones, which would cause an inevitable deconstruction of the cartilage and degeneration of the carpus. To avoid unnecessary friction between the palmar bone and the liner, the contact area of the liner was designed based on the articular surface of the contralateral side distal radius using Mimics software. In addition, optimization depended on the shape of the carpus of the affected side was performed. Therefore, cartilage degeneration and radio carpus osteoarthritis could be effectively prevented with a best-fit contact area. Second, the thicker the polyethylene liner is, the more easily it can detach from the metal portion, due to the lever principle. The thicker liner used in previous studies ${ }^{13,14}$ created an increased potential risk for liner dislocation. Considering that the wrist is a nonweight-bearing joint, we suggest that a $0.5-1-\mathrm{cm}$-thick liner is a better choice. We also designed a taper column with buckles to avoid dislocation of the liner. Third, we reserved the bone crest of the distal radius on the shaft to ensure proper implantation. This remaining crest of the shaft could act as a guideline to implant because of the crest continuity. Implanting the prosthesis with no rotation would result in a satisfactory ROM. Another detail for design of the shaft is that the length of the prosthesis must be suitable to the tumorous defect to avoid dislocation. We measured precisely by using 3D-CT scanning data that were imported into the Mimics software. The length of the tumorous defect was defined as the vertical distance between the ulnar head and a safe margin. The surgeons could easily locate the osteotomy plane using this definition during the surgery. Finally, joint restoration is essential to decrease the frequency of dislocation of the prosthesis. We designed seven pores distributed along the edge of the contact surface to fix the radio carpus joint more stably, whereas previous studies ${ }^{13-15}$ used fewer pores to fix the carpus joint. For repair of the distal radioulnar joint, three pores must be placed longitudinally in the upper middle portion of the joint surface to ensure that the distal ulnar is fixed in the middle position of the joint to allow more flexible supination and pronation.

Proper wrist joint fixation is one of the most demanding procedures during surgery to achieve satisfactory flexibility and stability. However, it is also a priority for prosthetic replacement. Adjusting the suturing tension and repairing the joint capsule sequentially are two main aspects to be considered for achieving better ROM and a more stable joint. First, we adjusted the tension by the positioning of the wrist joint when it was sutured. 


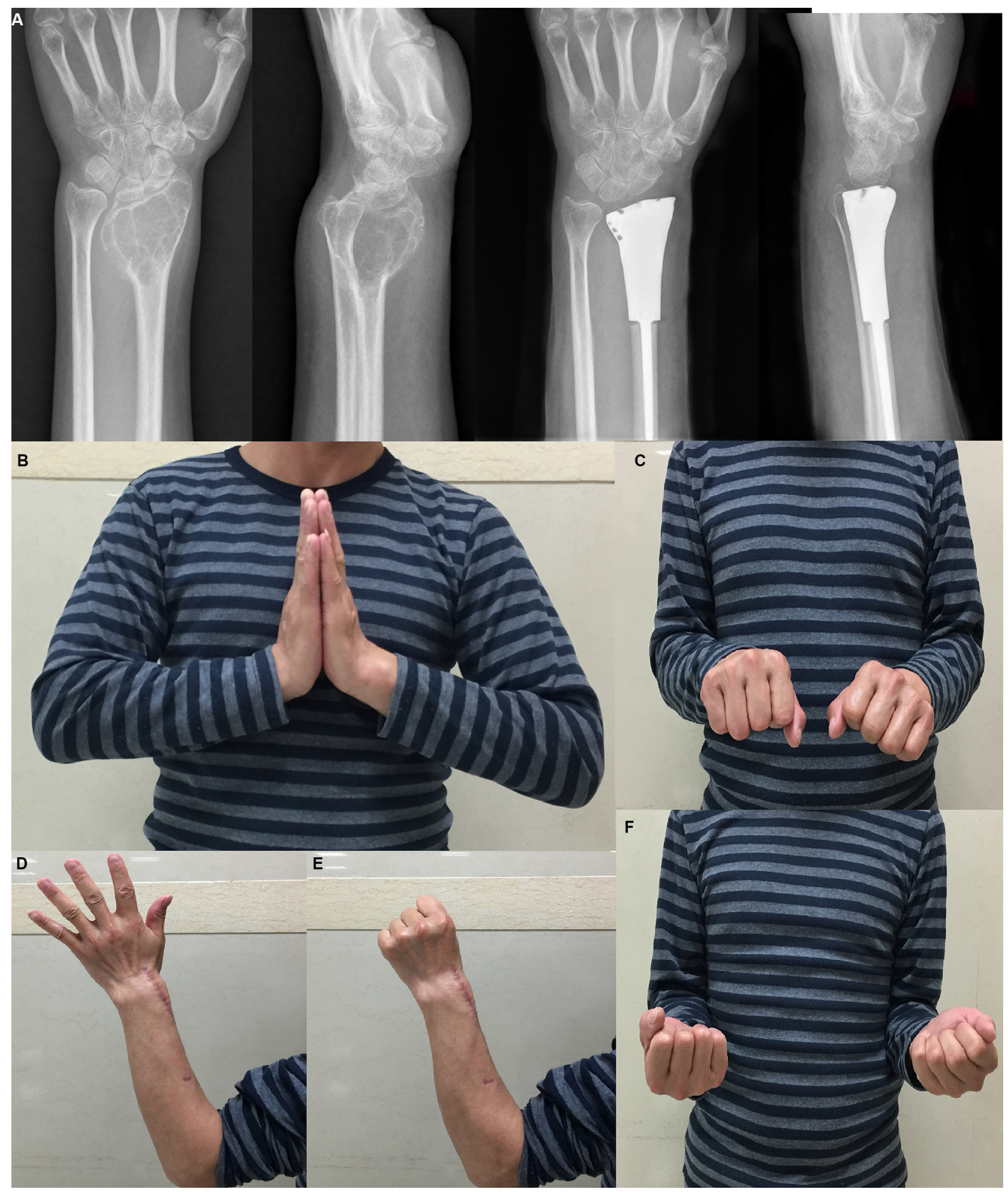

Figure 8 A 46-year-old man $\sim 18$ months after the resection of Campanacci grade III GCTs of the distal radius with 3D prosthetic reconstruction.

Notes: (A) Radiographic comparison between preoperative joint and postoperative joint. (B) Dorsiflexion of affected side could reach $70^{\circ}$ after the surgery. (C and $\mathbf{F}$ ) Pronation and supination were good. (D and E) Movement and sensation of finger and thumb were satisfactory.

Abbreviations: 3D, three-dimensional; GCT, giant cell tumor.

We suggest that $10^{\circ}-15^{\circ}$ of extension is the optimal position for the mobility of dorsiflexion when suturing the dorsal side. However, the palmar flexion was partially sacrificed to enhance this stability. Second, suturing the retained tissues to the pores in the prosthesis in a proper order makes joint repair easier and more efficient. We suggest that the palm ulnar side should be 


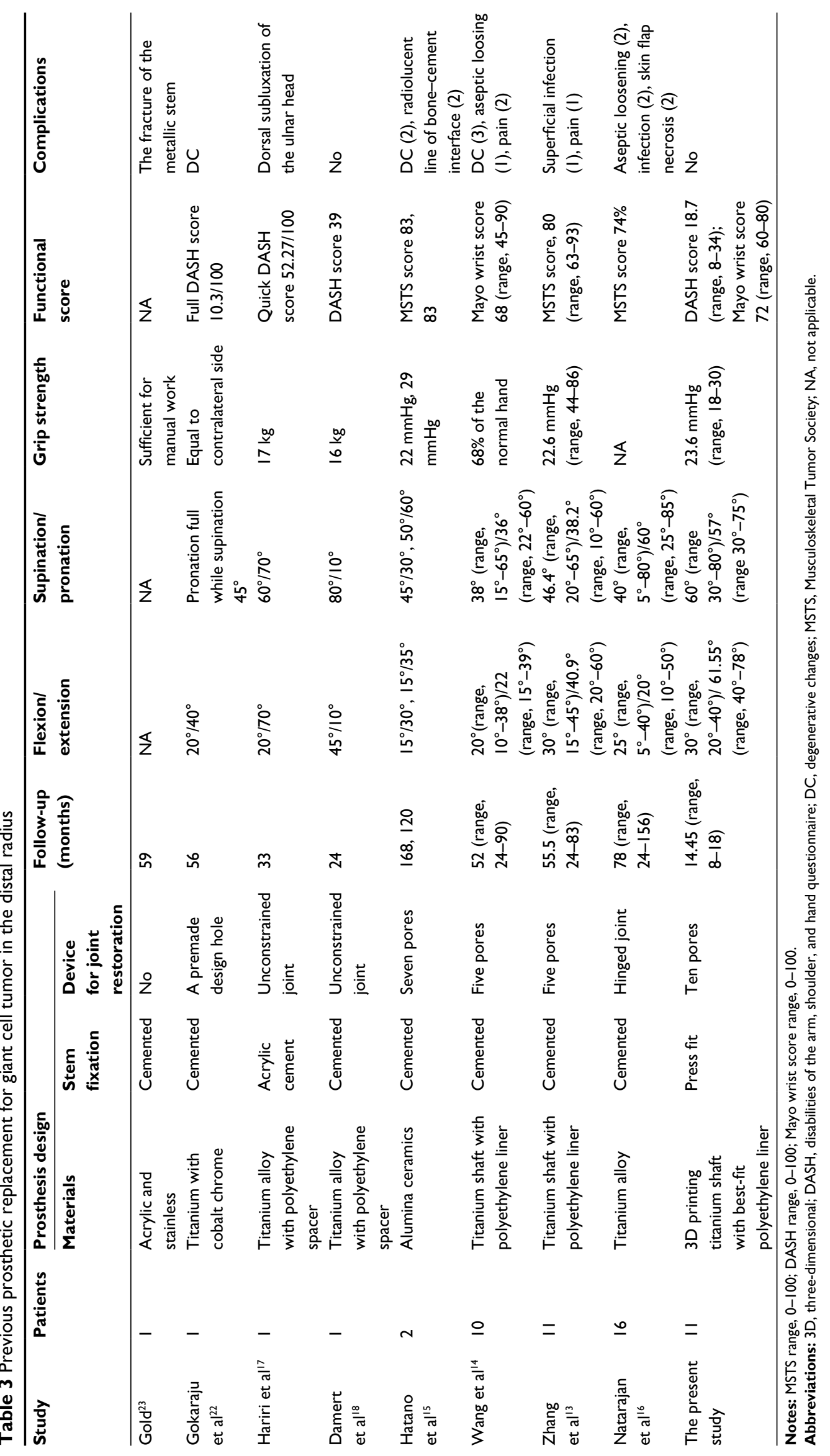


the first sewing point. The palm radial side, dorsal radial side, and dorsal ulnar side are then sequentially sutured.

We recognized the following limitations of our results. The duration of the follow-up is not, yet, sufficient to verify the long-term efficacy of this new design prosthesis and promising surgical techniques. In addition, it is also possible that more complications or problems might arise as we follow these patients for a longer time. The small number of patients is another limitation, and we also had no control group; however, Campanacci grade III GCTs are rare. Therefore, a larger multi-institutional study is needed to ideally compare this approach with other types of reconstruction.

\section{Conclusion}

The use of uncemented 3D-printed prosthetic reconstruction can be an alternative option for Campanacci grade III or recurrent GCTs of the distal radius after en bloc resection. The ability to perfectly imitate the contralateral normal shape of the wrist, the best-fit joint surface, press-fit fixation, and careful reconstruction of the soft tissue around the distal ulnoradial joint and the radiocarpal joint lead to reasonable postoperative wrist function and cartilage protection. As we have collected only short-term follow-up results, the long-term efficacy of local controls for GCTs and postoperative wrist motion are yet to be observed.

\section{Data sharing statement}

The data sets supporting the conclusions of this article are included within the article.

\section{Acknowledgments}

This work was supported, in part, by the National Natural Science Foundation of China (81702664) and the Department of Science and Technology of Sichuan Province (2014SZ0209).

\section{Author contributions}

MX Lu, M Li, YJ Li, and CQ Tu were involved with the concept and design of this manuscript. Y Luo and WL Zhang were involved with the acquisition of subjects and data. MX Lu and CQ Tu were involved in the design of the prosthesis. WL Zhang and CQ Tu were involved in postsurgical evaluation of the patients. All authors contributed toward data analysis, drafting and critically revising the paper, gave final approval of the version to be published, and agree to be accountable for all aspects of the work.

\section{Disclosure}

The authors report no conflicts of interest in this work.

\section{References}

1. Amanatullah DF, Clark TR, Lopez MJ, Borys D, Tamurian RM. Giant cell tumor of bone. Orthopedics. 2014;37(2):112-120.

2. Raskin KA, Schwab JH, Mankin HJ, Springfield DS, Hornicek FJ. Giant cell tumor of bone. J Am Acad Orthop Surg. 2013;21(2):118-126.

3. Errani C, Ruggieri P, Asenzio MA, et al. Giant cell tumor of the extremity: a review of 349 cases from a single institution. Cancer Treat Rev. 2010;36(1):1-7.

4. Campanacci M, Baldini N, Boriani S, Sudanese A. Giant-cell tumor of bone. J Bone Joint Surg Am. 1987;69(1):106-114.

5. Pazionis TJ, Alradwan H, Deheshi BM, Turcotte R, Farrokhyar F, Ghert M. A systematic review and meta-analysis of en-bloc vs intralesional resection for giant cell tumor of bone of the distal radius. Open Orthop J. 2013;7:103-108.

6. Goldenberg RR, Campbell CJ, Bonfiglio M. Giant-cell tumor of bone. An analysis of two hundred and eighteen cases. J Bone Joint Surg Am. 1970;52(4):619-664.

7. McDonald DJ, Sim FH, McLeod RA, Dahlin DC. Giant-cell tumor of bone. J Bone Joint Surg Am. 1986;68(2):235-242.

8. Harness NG, Mankin HJ. Giant-cell tumor of the distal forearm. J Hand Surg Am. 2004;29(2):188-193.

9. Salunke AA, Shah J, Warikoo V, et al. Giant cell tumor of distal radius treated with ulnar translocation and wrist arthrodesis. J Orthop Surg (Hong Kong). 2017;25(1):2309499016684972.

10. Chadha M, Arora SS, Singh AP, Gulati D, Singh AP. Autogenous nonvascularized fibula for treatment of giant cell tumor of distal end radius. Arch Orthop Trauma Surg. 2010;130(12):1467-1473.

11. Muramatsu K, Ihara K, Azuma E, et al. Free vascularized fibula grafting for reconstruction of the wrist following wide tumor excision. Microsurgery. 2005;25(2):101-106.

12. Szabo RM, Anderson KA, Chen JL. Functional outcome of en bloc excision and osteoarticular allograft replacement with the Sauve-Kapandji procedure for Campanacci grade 3 giant-cell tumor of the distal radius. J Hand Surg Am. 2006;31(8):1340-1348.

13. Zhang S, Xu MT, Wang XQ, Wang JJ. Functional outcome of en bloc excision and custom prosthetic replacement for giant cell tumor of the distal radius. J Orthop Sci. 2015;20(6):1090-1097.

14. Wang B, Wu Q, Liu J, Chen S, Zhang Z, Shao Z. What are the functional results, complications, and outcomes of using a custom unipolar wrist hemiarthroplasty for treatment of grade III giant cell tumors of the distal radius? Clin Orthop Relat Res. 2016;474(12):2583-2590.

15. Hatano H, Morita T, Kobayashi H, Otsuka H. A ceramic prosthesis for the treatment of tumours of the distal radius. J Bone Joint Surg Br. 2006;88(12):1656-1658.

16. Natarajan MV, Chandra Bose J, Viswanath J, Balasubramanian N, Sameer M. Custom prosthetic replacement for distal radial tumours. Int Orthop. 2009;33(4):1081-1084.

17. Hariri A, Facca S, Di Marco A, Liverneaux P. Massive wrist prosthesis for giant cell tumour of the distal radius: a case report with a 3-year follow-up. Orthop Traumatol Surg Res. 2013;99(5):635-638.

18. Damert HG, Altmann S, Kraus A. Custom-made wrist prosthesis in a patient with giant cell tumor of the distal radius. Arch Orthop Trauma Surg. 2013;133(5):713-719.

19. Saraf SK, Goel SC. Complications of resection and reconstruction in giant cell tumour of distal end of radius - an analysis. Ind J Orthop. 2005;39(4):206-211.

20. Tolstopiatov BA, Korolev VI, Borisiuk BE. [Surgical treatment of giant cell tumor of the distal end of the radius]. Ortop Travmatol Protez. 1979;(3):42-44. Russian.

21. Taylor GI, Corlett RJ, Ashton MW. The evolution of free vascularized bone transfer: a 40-year experience. Plast Reconstr Surg. 2016;137(4): 1292-1305.

22. Gokaraju K, Sri-Ram K, Donaldson J, et al. Use of a distal radius endoprosthesis following resection of a bone tumour: a case report. Sarcoma. 2009;2009:938295. 
23. Gold AM. Use of a prosthesis for the distal portion of the radius following resection of a recurrent giant-cell tumor. J Bone Joint Surg Am 1957;39-A(6):1374-1380.

24. Hudak PL, Amadio PC, Bombardier C. Development of an upper extremity outcome measure: the DASH (disabilities of the arm, shoulder and hand) [corrected]. The Upper Extremity Collaborative Group (UECG). Am J Ind Med. 1996;29(6):602-608.
25. Amadio PC, Berquist TH, Smith DK, Ilstrup DM, Cooney WP 3rd, Linscheid RL. Scaphoid malunion. J Hand Surg Am. 1989;14(4): 679-687.

26. Cheng CY, Shih HN, Hsu KY, Hsu RW. Treatment of giant cell tumor of the distal radius. Clin Orthop Relat Res. 2001;(383): 221-228.
Cancer Management and Research is an international, peer-reviewed open access journal focusing on cancer research and the optimal use of preventative and integrated treatment interventions to achieve improved outcomes, enhanced survival and quality of life for the cancer patient. The manuscript management system is completely online and includes 